\title{
Some Physical Region Mass Shell Properties of Renormalized Feynman Integrals
}

\author{
Colston Chandler \\ Seminar für theoretische Physik der Eidgenössischen Technischen Hochschule, Zürich
}

Received June 8, 1970

\begin{abstract}
Certain properties proved in $S$-matrix theory on the basis of macroscopic causality are verified for renormalized Feynman integrals. The technique uses the analytic renormalization of Speer and a specific distortion of the contour of integration. It is proved for an arbitrary Feynman graph $G$ that the corresponding renormalized Feynman integral is holomorphic in the (mass shell) physical region except on the positive- $\alpha$ Landau surface $\mathfrak{L}^{+}\{G\}$. Under a certain assumption about the geometry of $\mathfrak{L}^{+}\{G\}$ an "ic-prescription" is constructed for continuing (in the mass shell) around $\mathfrak{I}^{+}\{G\}$. The difficulties involved in removing this assumption are discussed.
\end{abstract}

\section{Introduction}

Feynman integrals have been an important source of understanding of analytic properties of scattering functions in $S$-matrix theories $[1,2]$. Unfortunately, previous study of the mass shell properties of these integrals has lacked generality because no method was known that dealt efficiently enough with the problems of renormalization. This deficiency can now be repaired with the recently developed techniques of analytic renormalization [3-6].

The object of this work is the verification for renormalized integrals of mass shell properties analogous to those derived for $S$-matrix elements on the basis of macroscopic causality $[7,8]$. The first of these $S$-matrix properties is that the scattering functions are holomorphic at points of the physical region that are not on some positive- $\alpha$ Landau surface. The second property concerns points on such surfaces. At these points a scattering function can be written as a sum of terms, each of which is the boundary value of a holomorphic function. Usually, only one boundary value term is necessary, but there do exist exceptional points at which two or more are needed [9]. In such situations there is a question of how the singularities are distributed among the various boundary value terms. This is essentially resolved by assumption, the assumption being called the "independence property" [10]. The derivation of this property in the context of Feynman integrals is a second, but presently unrealized, goal of this work. 
In Section II the analytic renormalization of Speer [3-5] is sketched and a definition of renormalized Feynman integrals given. Section III contains preliminary results related to the technique of contour deformation used in later sections. Section IV is devoted to a proof that a renormalized Feynman integral is holomorphic at all points of the physical region that are not on the appropriate positive- $\alpha$ Landau surfaces. An

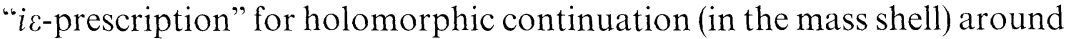
the positive- $\alpha$ Landau surface is derived in Section V. The comments on the independence property are found there, as is a correction to the proof of Theorem 13 of [7].

\section{Analytic Renormalization}

\section{A. Feynman Graphs}

A connected Feynman graph is a set of vertices connected by directed line segments. The vertices are represented by an index set $\mathscr{V}=\{0,1, \ldots, V\}$. One of these vertices, say the zeroth, is specified as a reference vertex, and the remaining vertices are labelled by the reduced set $\mathscr{V}_{0}=\mathscr{V}-\{0\}$. The lines are represented by an index set $\mathscr{L}=\{1,2, \ldots, L\}$, and their orientation by an incidence matrix $[e]$,

$$
\begin{aligned}
e(r, l) & =+1 \text { if line } l \in \mathscr{L} \text { is directed into vertex } r \in \mathscr{V}, \\
& =-1 \text { if line } l \text { is directed out of } r, \\
& =0 \text { otherwise. }
\end{aligned}
$$

The assumption that $G$ is connected means that $\operatorname{rank}[e]=V$.

Each line $l$ of $G$ is associated with a "particle" of (possibly complex) momentum $p_{l}{ }^{1}$, (real) mass $\mu_{l}$, and spin $\sigma_{l}$.

There are also external particles associated with the vertices of the graph $G$. They are indexed by the set $\mathscr{L}_{0}=\{1,2, \ldots, n\}$, and their distribution among the vertices is described by a matrix $[f]$,

$$
\begin{aligned}
f(r, j) & =+1 \text { if particle } j \in \mathscr{L}_{0} \text { is incident on vertex } r \in \mathscr{W}, \\
& =0 \text { otherwise. }
\end{aligned}
$$

It is assumed that at least one external particle is incident on the reference vertex. Each particle has a (possibly complex) momentum $k_{j}$ and (real) non-zero mass $m_{j}$. The spin is of no interest here. The total external momentum at each vertex is

$$
q_{r}(K)=\sum_{\mathscr{L}_{0}} f(r, j) k_{j}, \quad(r \in \mathscr{V}),
$$

${ }^{1}$ Momentum always means four-momentum. The metric is always

$$
g_{00}=-g_{11}=-g_{22}=-g_{33}=1 \text {. }
$$


and it is assumed that momentum is conserved at each vertex,

$$
\sum_{\mathscr{L}} e(r, l) p_{l}+q_{r}(K)=0, \quad(r \in \mathscr{V}) .
$$

(Other conservation laws are unimportant for this paper.) Eq. (2.4) leads to the requirement that $K=\left(k_{1}, \ldots, k_{n}\right)$ belong to the complex manifold

$$
\mathfrak{E}=\left\{K \mid K=\left(k_{1}, \ldots, k_{n}\right), \sum k_{j}=0\right\} .
$$

The real section of $\mathfrak{E}$ is denoted by $\mathbb{E}$. The complex mass shell of the external particles is ${ }^{2}$

$$
\mathfrak{M}=\left\{K \in \mathbb{E} \mid k_{j}^{2}=m_{j}^{2}, j \in \mathscr{L}_{0}\right\},
$$

and the set of manifold points of $\mathfrak{M}$ is denoted by $\mathfrak{B}$. A physical region $\mathfrak{B}$ is a real section of $\mathfrak{M}$, less those points where two (or more) negativeenergy momenta or two (or more) positive-energy momenta are parallel. The negative-energy particles are called the final particles, and the others initial particles.

It is useful to introduce the notion of a stable particle graph on the physical region $\mathfrak{P}$. At each vertex there should be at least two incoming particles and two outgoing particles. For internal particles the incidence matrix $[e]$ specifies which are incoming $(e(r, l)$ positive) and which are outgoing $(e(r, l)$ negative). For the external particles this is done by the incidence matrix $e_{0}(r, j)=\left(k_{j}^{0} /\left|k_{j}^{0}\right|\right) f(r, j)$. A graph $G$ is then a stable particle graph on $\mathfrak{P}$ if the total incidence matrix $\left[e, e_{0}\right]$ has in each row at least two positive and two negative entries.

Finally, a contraction $G^{\prime}$ of $G$, written $G^{\prime} \subseteq G$ is a Feynman graph with the following properties. The set $\mathscr{L}^{\prime}$ is nonempty. There are a surjection $\pi: \mathscr{V} \rightarrow \mathscr{V}^{\prime}$, an injection $i: \mathscr{L}^{\prime} \rightarrow \mathscr{L}$, and a bijection $i_{0}: \mathscr{L}_{0}^{\prime} \rightarrow \mathscr{L}_{0}$. The incidence matrices are related by $e^{\prime}\left(\pi(r), l^{\prime}\right)=e\left(r, i\left(l^{\prime}\right)\right), f^{\prime}\left(\pi(r), j^{\prime}\right)$ $=f\left(r, i_{0}\left(j^{\prime}\right)\right)$. The particles associated with the lines of $G^{\prime}$ are the same as those associated with the corresponding lines of $G$. The graph $G^{\prime}$ is a proper contraction of $G, G^{\prime} \subset G$, if $i\left(\mathscr{L}^{\prime}\right) \neq \mathscr{L}$. The set of all graphs that are contractions either of $G$, or of a graph formed from $G$ by changing the directions of some of the lines, is denoted by $\{G\}$.

\section{B. Feynman Integrals}

The analytic renormalization described here is that of Speer [3-5].

The first step in this scheme is to write the Feynman integral for the connected graph $G$ in the usual way:

$$
\int \prod_{\mathscr{L}}\left[d^{4} p_{l} \Delta_{l, F}\left(p_{l}\right)\right] \prod_{\mathscr{V}_{0}} \delta\left(q_{r}(K)+\sum_{\mathscr{L}} e(r, l) p_{l}\right),
$$

${ }^{2}$ Units $\hbar=c=1$ are adopted. 
where all momenta are understood to be real. Then the Feynman propagators are replaced by

$$
\Delta_{l}\left(\lambda_{l}, p_{l}\right)=\Delta_{l, F}\left(p_{l}\right)\left[(-i)\left(p_{l}^{2}-\mu_{l}^{2}+i \varepsilon\right)\right]^{1-\lambda_{l},}, \quad(l \in \mathscr{L}),
$$

where the $\lambda_{l}$ are complex. The resultant integral, when expressed as an integral over the Feynman parameters $\alpha=\left(\alpha_{1}, \ldots, \alpha_{L}\right)$, has the form

$$
\begin{gathered}
F_{\varepsilon}(\lambda, K)=\lim _{r \rightarrow 0^{+}} \lim _{R \rightarrow \infty} F_{\varepsilon, r, R}(\lambda, K), \\
F_{\varepsilon, r, R}(\lambda, K)=\int_{r}^{R} d \alpha Z(\alpha, K) W(\alpha, \lambda) \exp \left[i Y(\alpha, K)-\varepsilon \sum \alpha_{l}\right] .
\end{gathered}
$$

The function $Z(\alpha, K)$ is related to the spins of the particles and is a polynomial in the variables $\alpha_{l}, l \in \mathscr{L}$, and $q_{r}(K), r \in \mathscr{V}_{0}$. The function $W(\alpha, \lambda)$ is defined by

$$
W(\alpha, \lambda)=C(\alpha)^{-\sigma-2} \prod_{\mathscr{L}}\left[\alpha_{l}^{\lambda_{l}-1} \Gamma\left(\lambda_{l}\right)^{-1}\right],
$$

where $\sigma=2 \sum \sigma_{l}$ and where

$$
\begin{gathered}
C(\alpha)=\left(\prod_{\mathscr{L}} \alpha_{l}\right) \operatorname{det}[E(\alpha)], \\
E_{r s}(\alpha)=\sum_{\mathscr{L}} e(r, l) \alpha_{l}^{-1} e(s, l), \quad\left(r, s \in \mathscr{V}_{0}\right) .
\end{gathered}
$$

The function $Y(\alpha, K)$ is defined by

$$
\begin{gathered}
Y(\alpha, K)=\sum_{\mathscr{L}} \alpha_{l}\left(r_{l}^{2}-\mu_{l}^{2}\right), \\
r_{l}(\alpha, K)=-\alpha_{l}^{-1} \sum_{r, s \in \mathscr{V}_{0}} e(r, l) E_{r s}^{-1}(\alpha) q_{s}(K) .
\end{gathered}
$$

The second step in renormalization is meromorphic continuation of $F_{\varepsilon}(\lambda, K)$ in $\lambda$. For complex $\lambda$ in the set

$$
\begin{aligned}
\Lambda & =\left\{\lambda \in \mathbb{C}^{L} \mid \operatorname{Re} \lambda_{l}>\lambda_{0}, 1 \leqq l \leqq L\right\}, \\
\lambda_{0} & =(\sigma+2)(L-V+1),
\end{aligned}
$$

and for real $K$ the limit (2.9) can be taken, convergence of the integral being absolute. The integral $F_{\varepsilon}(\lambda, K)$ is holomorphic on $A$ and $C^{\infty}$ on $\mathbb{E}$. The limit $\varepsilon \rightarrow 0^{+}$yields an object $F(\lambda, K)$ that is holomorphic on $\Lambda$ and a Schwartz distribution on $\mathbb{E}^{r}$. Finally, the distribution

$$
G(\lambda, K)=F(\lambda, K) \prod_{\mathscr{M} \subseteq \mathscr{L}} \Gamma\left(\sum_{\mathscr{M}}\left(\lambda_{l}-\lambda_{0}\right)\right)^{-1},
$$

where the product is over all subsets $\mathscr{M}$ of $\mathscr{L}$, can be continued holomorphically from $\Lambda$ to all of $\mathbb{C}^{L}$. 
This enlarged domain of holomorphy of $F(\lambda, K)$ permits the following contour integration

$$
F(K)=(L !)^{-1}(2 \pi i)^{-L} \sum_{P} \int_{\gamma(P)} d \lambda F(\lambda, K) \prod_{\mathscr{L}}\left(\lambda_{l}-1\right)^{-1} .
$$

The summation in (2.17) is over all permutations $P$ of $\mathscr{L}$,

$$
P(\mathscr{L})=\{P(1), \ldots, P(L)\} .
$$

The contour $\gamma(P)$ is given by the counterclock wise contours $\left|\lambda_{l}-1\right|=\gamma_{P(l)}$, where $\gamma_{L}$ is sufficiently small and $\gamma_{l}>\sum_{m=1}^{l-1} \gamma_{m}$ for all $l$. The remarkable feature of (2.17) is that it defines a standard Bogoliubov-Parasuik-Hepp additive renormalization $[3-5,11]$. Hence, it is taken as the definition of the renormalized Feynman integral corresponding to the graph $G$.

\section{Contour Deformation}

The principal tool of this paper is the standard one of deformation of the contour of $\alpha$-integration. Perhaps the most elegant way to go about this is with general homological theorems $[12,13]$. This approach is, however, not adopted here because of the detailed information about the contour deformation that is needed in Section V. Instead, a more pedestrian constructive approach is used.

It is convenient to begin by proving three lemmata. The first is a slight generalization of a result of Speer (Lemma 1 of [3], Lemma 3.7 of [4]).

Lemma 1. Let $G$ be a Feynman graph, and let $P$ be a permutation of $\mathscr{L}$. Let $G_{P, L}$ be the subgraph of $G$ formed by the internal lines $P(1)$ through $P(l)$ and their vertices. Let $N_{P, l}$ be the number of independent loops of $G_{P, l}$. Finally, let $z=\left(z_{1}, \ldots, z_{L}\right)$ have the form

$$
z_{P(l)}=t_{L} t_{L-1} \ldots t_{l} \hat{z}_{P(l)}, \quad(l \in \mathscr{L}) .
$$

Then the following equations, where $\hat{t}=\left(t_{L-1}, \ldots, t_{1}\right)$ and $\hat{z}=\left(\hat{z}_{L}, \ldots, \hat{z}_{1}\right)$, are true:

$$
\begin{aligned}
C(z) & =\left(\prod_{\mathscr{L}} t_{l}^{N_{P, l}}\right) C_{P}(\hat{t}, \hat{z}) ; \\
z_{l}^{-1} \sum_{s \in \mathscr{V}_{0}} e(s, l) E_{r s}^{-1}(z) & =\hat{z}_{l}^{-1} C_{P}^{-1} D_{l r}^{P}(\hat{t}, \hat{z}), \quad\left(l \in \mathscr{L}, r \in \mathscr{V}_{0}\right) .
\end{aligned}
$$

The functions $C_{P}$ and $D_{l r}^{P}$ are polynomials, and the polynomial $C_{P}$ cannot vanish on any compact subset of

$$
\left\{(\hat{t}, \hat{z}) \in \mathbb{R}^{L-1} \otimes \mathbb{C}^{L} \mid \hat{t}_{l} \geqq 0,1 \leqq l \leqq L-1 ; \operatorname{Re} \hat{z}_{m}>0,1 \leqq m \leqq L\right\} .
$$


Proof. The only part of Speer's discussion that needs modification is the proof that $C_{P}$ cannot vanish on compact subsets of (3.4). Suppose otherwise, that $C_{P}$ does vanish at $(\hat{t}, \hat{z})$. Then the matrix

$$
\left(t_{L} \ldots t_{l_{1}}\right)^{-\frac{1}{2}}\left(t_{L} \ldots t_{l,}\right)^{-\frac{1}{2}} \sum_{\mathscr{L}} z_{l} \eta_{l i} \eta_{l j}, \quad(1 \leqq i, j \leqq L-V),
$$

which has determinant $C_{P}$, must be singular. As in [3] the indices $l_{i}$ correspond to those values of $l$ for which $N_{P, l}=1+N_{P, l-1}$. The matrix $[\eta]$ is the loop matrix of $G$; it can be any $L \times(L-V)$ matrix, the columns of which span the null space of the incidence matrix $[e]$. Here it is chosen to have the additional property that $\eta_{P(l) i}=0$ if $l>l_{i}$. If the matrix (3.5) is singular, there is a nonzero eigenvector $v$ with zero eigenvalue. Multiplying (3.5) from the right by $v$ and from the left by its complex conjugate $v^{*}$ yields

$$
\sum_{\mathscr{L}} \hat{z}_{P(l)}\left|\sum_{i} \eta_{P(l) i} v_{i}\left(t_{L} \ldots t_{l}\right)^{\frac{1}{2}}\left(t_{L} \ldots t_{l_{l}}\right)^{-\frac{1}{2}}\right|^{2}=0
$$

Since $\operatorname{Re} \hat{z}_{l}>0$ for all $l$, each term in (3.7) must vanish. This is possible only if $v$ vanishes [3], contrary to hypothesis. Hence the matrix (3.5) is non-singular and $C_{P}$ does not vanish. q.e.d.

The second lemma is more directly concerned with the manner in which the contour deformation is to be done. Let $A$ denote the set

$$
A=\left\{\alpha \in \mathbb{R}^{L} \mid \alpha_{l}>0,1 \leqq l \leqq L\right\},
$$

and let $A^{P}$, where $P$ is a permutation of $\mathscr{L}$, denote

$$
A^{P}=\left\{\alpha \in A \mid \alpha_{P(1)} \leqq \alpha_{P(2)} \leqq \cdots \leqq \alpha_{P(L)}\right\} .
$$

Sector coordinates on $A^{P}$ are defined by

$$
t_{L} t_{L-1} \ldots t_{l}=\alpha_{P(l)}, \quad(l \in \mathscr{L}) .
$$

The fact that $\alpha$ belongs to $A^{P}$ means, in particular, that $\hat{t}=\left(t_{L-1}, \ldots, t_{1}\right)$ is restricted to the set

$$
\hat{T}=\left\{\hat{t} \in \mathbb{R}^{L-1} \mid 0 \leqq \hat{t}_{l} \leqq 1,1 \leqq l \leqq L-1\right\} .
$$

Finally, define a one parameter family of mappings $\zeta^{x}: A \otimes \mathfrak{E} \rightarrow \mathbb{C}^{L}$ by

$$
\begin{aligned}
& \zeta_{l}^{x}(\alpha, K)=\alpha_{l} \hat{\zeta}_{l}^{x}(\alpha, K), \\
& \hat{\zeta}_{l}^{x}(\alpha, K)=\exp \left\{i x\left(r_{l}^{2}-\mu_{l}^{2}\right)\right\}, \quad(l \in \mathscr{L}) .
\end{aligned}
$$

The parameter $x$ is real, and the vectors $r_{l}$ are given by (2.14).

Lemma 2. Let the notation of Lemma 1 be adopted. Let $\alpha$ be restricted to $A^{P}$, and let sector coordinates (3.9) be introduced. Let $\boldsymbol{\aleph}_{0} \subset$ 这 be a compact convex set. Then there exists $\bar{x}>0$ such that the following statements are true: 
(i) The functions $\hat{\zeta}_{l}^{x}$ are holomorphic in all variables $(x, \hat{t}, K)$ on $[0, \bar{x}] \otimes \hat{T} \otimes \mathfrak{S}_{0}{ }^{3}$. They do not depend on $t_{L}$.

(ii) The equalities

$$
\begin{gathered}
C\left(\zeta^{x}\right)=\left(\prod_{\mathscr{L}^{\prime}} t_{l}^{N_{P}, l}\right) \tilde{C}_{P}(x, \hat{t}, K), \\
\left(\zeta_{l}^{x}\right)^{-1} \sum_{s \in \mathscr{V}_{0}} e(s, l) E_{r s}^{-1}\left(\zeta^{x}\right)=\left(\hat{\zeta}_{l}^{x}\right)^{-1} \tilde{C}_{P}^{-1} \tilde{D}_{l r}^{P}(x, \hat{t}, K),
\end{gathered}
$$

are true. The functions $\tilde{C}_{P}$ and $\tilde{D}_{l r}^{P}$ are holomorphic in $(x, \hat{t}, K)$, and $\tilde{C}_{P}$ does not vanish, on $[0, \bar{x}] \otimes \hat{T} \otimes \mathfrak{R}_{0}$.

Proof. Part (i) is immediate from (2.14) and Lemma 1. In particular the functions $r_{l}^{2}-\mu_{l}^{2}$ are bounded on $\hat{T} \otimes \mathfrak{R}_{0}$. Consequently, the number $\bar{x}$ can be chosen small enough that $\operatorname{Re} \hat{\zeta}_{l}^{x}>0$ on $[0, \bar{x}] \otimes \hat{T} \otimes \mathfrak{R}_{0}$. Substitution of $\hat{\zeta}_{l}^{x}$ for $\hat{z}_{l}$ in Lemma 1 completes the proof. q.e.d.

The final lemma is primarily concerned with the function $\tilde{Y}(x, \alpha, K)$ $=Y\left(\zeta^{x}(\alpha, K), K\right)$ on $\bar{A} \otimes \mathfrak{R}_{0}$, where $\bar{A}$ is the closure of $A$.

Lemma 3. There exists $\bar{x}>0$ such that for each $x \in[0, \bar{x}]$ the mapping $\zeta^{x}$ can be continuously extended from $A \otimes \mathfrak{R}_{0}$ to $\bar{A} \otimes \mathfrak{S}_{0}$. In addition, the following statements are true for all $x \in[0, \bar{x}]$.

(i) Let $\alpha$ and $\alpha^{\prime}$ be in $\bar{A}, K$ and $K^{\prime}$ in $\Re_{0}$, and let ${ }^{4}$

$$
n\left(\alpha, \alpha^{\prime} ; K, K^{\prime}\right)=\left|\alpha-\alpha^{\prime}\right|+\left\|K-K^{\prime}\right\| \max \left\{|\alpha|,\left|\alpha^{\prime}\right|\right\} .
$$

Then there exists a finite number $M$, dependent only on $\bar{x}$ and $\mathfrak{\Re}_{0}$, such that

$$
\begin{aligned}
\left|\zeta^{x}(\alpha, K)-\zeta^{x}\left(\alpha^{\prime}, K^{\prime}\right)\right| & \leqq M n\left(\alpha, \alpha^{\prime} ; K, K^{\prime}\right), \\
\left|\tilde{Y}(x, \alpha, K)-\tilde{Y}\left(x, \alpha^{\prime}, K^{\prime}\right)\right| & \leqq M n\left(\alpha, \alpha^{\prime} ; K, K^{\prime}\right), \\
\left\|V_{K} \tilde{Y}(x, \alpha, K)-\nabla_{K} \tilde{Y}\left(x, \alpha^{\prime}, K^{\prime}\right)\right\| & \leqq M n\left(\alpha, \alpha^{\prime} ; K, K^{\prime}\right) .
\end{aligned}
$$

(ii) For all $\alpha \in \bar{A}$ and $K \in\left(\mathfrak{R}_{0} \cap \mathbb{E}^{r}\right)$ the inequality

$$
\operatorname{Im} \tilde{Y}(x, \alpha, K) \geqq \frac{1}{2} x \sum_{\mathscr{L}} \alpha_{l}\left(r_{l}^{2}-\mu_{l}^{2}\right)^{2}
$$

holds.

Proof. Because $A$ is dense in $\bar{A}$ it is sufficient to prove the theorem for $\alpha$ and $\alpha^{\prime}$ in $A$. Consider first the mapping $\zeta^{x}$. Let $\alpha$ and $\alpha^{\prime}$ be any two points of $A$, and let $K$ and $K^{\prime}$ be any two points of $\mathfrak{K}_{0}$. Define

$$
\begin{aligned}
& \alpha_{y}=(1-y) \alpha+y \alpha^{\prime}, \\
& K_{y}=(1-y) K+y K^{\prime} .
\end{aligned}
$$

3 This is understood to mean that there exists an open set in $\mathbb{C} \otimes \mathbb{C}^{L-1} \otimes \mathfrak{E}$ containing $[0, \bar{x}] \otimes \hat{T} \otimes \Re_{0}$ on which the functions $\hat{\zeta}_{l}^{x}$ are holomorphic.

4 The norm $|\cdot|$ for numbers $z=\left(z_{1}, \ldots, z_{L}\right) \in \mathbb{C}^{L}$ is $|z|=\left(\sum\left|z_{m}\right|^{2}\right)^{1 / 2}$. The norm $\|\cdot\|$ for $n$-tuples $U=\left(u_{1}, \ldots u_{n}\right)$ of complex-valued four vectors is $\|U\|=\left(\sum_{J, v}\left|\bar{u}_{, v}\right|^{2}\right)^{1 / 2}$. 
The mapping $\zeta^{x}\left(\alpha_{y}, K_{y}\right)$ is then $C^{\infty}$ in $y$ on the interval $[0,1]$. Therefore, the mean value theorem can be applied to each of the constituent real functions:

$\zeta^{x}(\alpha, K)-\zeta^{x}\left(\alpha^{\prime}, K^{\prime}\right)=\left(\alpha-\alpha^{\prime}\right) \cdot \nabla_{\alpha} \zeta^{x}\left(\alpha_{\bar{y}}, K_{\bar{y}}\right)+\left(K-K^{\prime}\right) \cdot \nabla_{K} \zeta^{x}\left(\alpha_{\bar{y}}, K_{\bar{y}}\right)$,

where $0 \leqq \bar{y} \leqq 1$. Eq. (3.20) is to be understood as a collection of real equations with perhaps a different $\bar{y}$ for each. Use of the Schwarz inequality yields

$$
\left|\zeta^{x}(\alpha, K)-\zeta^{x}\left(\alpha^{\prime}, K^{\prime}\right)\right| \leqq M n\left(\alpha, \alpha^{\prime} ; K, K^{\prime}\right), \quad\left(\alpha, \alpha^{\prime} \in A\right),
$$

where $M$ is the larger of

$$
\begin{aligned}
& M_{1}=\sup \left(\sum_{m, n}\left|\frac{\partial \zeta_{m}^{x}}{\partial \alpha_{n}}(\alpha, K)\right|^{2}\right)^{\frac{1}{2}}, \\
& M_{2}=\sup \left(\sum_{m}\left\|\Delta_{K} \zeta_{m}^{x}(\alpha, K)\right\|^{2}\right)^{\frac{1}{2}} .
\end{aligned}
$$

The supremum in (3.22) is over $x \in[0, \bar{x}], \alpha \in A, K \in \mathfrak{\Omega}_{0}$. That of (3.23) has the additional restriction $|\alpha| \leqq 1$ because of the factor $\max \left\{|\alpha|,\left|\alpha^{\prime}\right|\right\}$ in (3.14) and the homogeneity in $\alpha$ of $\zeta^{x}$.

Lemma 1 and Lemma 2 (i) are used to prove that $M_{1}$ and $M_{2}$ are finite. For example, the Jacobian in (3.22) is

$$
\begin{aligned}
& J_{m n}=\frac{\partial \zeta_{m}^{x}}{\partial \alpha_{n}} \\
& =\hat{\zeta}_{m}^{x}\left\{\delta_{m n}-2 i x r_{m} \cdot r_{n}\left[\delta_{m n}-\alpha_{n}^{-1} \sum_{r, s \in \mathscr{V}_{0}} e(r, m) E_{r s}^{-1}(\alpha) e(s, n)\right]\right\} .
\end{aligned}
$$

In each sector $A^{P}$, according to (2.14) and the lemmata, the functions $J_{m n}$ are holomorphic in the variables $(x, \hat{t}, K)$ on $[0, \hat{x}] \otimes \hat{T} \otimes \Omega_{0}$. They are therefore bounded on each set $[0, \bar{x}] \times A^{P} \otimes \mathfrak{\Re}_{0}$, implying that $M_{1}$ is finite. Finiteness of $M_{2}$ is proved in the same way.

The inequalities (3.16) and (3.17) are proved in exactly the same way, appeal being made to Lemma 2 (ii) rather than Lemma 1.

To prove (3.18) consider $\alpha \in A$ and $K \in\left(\mathfrak{\Re}_{0} \cap \mathbb{E}^{r}\right)$. Let $\bar{x}$ be chosen in agreement with Lemma 2. The function $\operatorname{Im} \tilde{Y}(x, \alpha, K)$ is $C^{\infty}$ in $x$ and can be expanded in a Taylor series with remainder:

$$
\operatorname{Im} \tilde{Y}(x, \alpha, K)=x \sum_{\mathscr{L}} \alpha_{l}\left(r_{l}^{2}-\mu_{l}^{2}\right)^{2}+\text { (remainder). }
$$

Explicit calculation, together with Lemma 2 and the Schwarz inequality, yields a bound for the remainder,

$$
\mid(\text { remainder }) \mid \leqq \frac{x^{2}}{2} \Delta \sum_{\mathscr{L}} \alpha_{l}\left(r_{l}^{2}-\mu_{l}^{2}\right)^{2},
$$


in which $\Delta$ depends only on $\bar{x}$ and $\boldsymbol{S}_{0}$. Inequality (3.18) results from choosing a new $\bar{x}$ as the lesser of the original $\bar{x}$ and $(\Delta)^{-1}$. q.e.d.

The manner in which the contour of $\alpha$-integration is to be deformed can now be formulated as a theorem.

Theorem 1. Let $\Omega^{r} \subset \mathbb{E}^{r}$ be a compact convex set, and let $\Lambda_{0} \subset \Lambda$ be compact. Then there exists $\bar{x}>0$ such that for all $\varepsilon>0$ the limit

$$
\begin{gathered}
F_{\varepsilon}(\lambda, K)=\lim _{r \rightarrow 0^{+}} \lim _{R \rightarrow \infty} \tilde{F}_{\varepsilon, r, R}(\lambda, K), \\
\tilde{F}_{\varepsilon, \boldsymbol{r}, R}(\lambda, K)=\int_{r}^{R} d \alpha \frac{\partial\left(\zeta^{x}\right)}{\partial(\alpha)} Z\left(\zeta^{x}, K\right) W\left(\zeta^{x}, \lambda\right) \exp \left[i Y\left(\zeta^{x}, K\right)-\varepsilon \sum_{\mathscr{L}} \zeta_{l}^{x}\right],
\end{gathered}
$$

is obtained uniformly in $(x, \lambda, K)$ on $(0, \bar{x}] \otimes \Lambda_{0} \otimes \Omega^{r}$. The convergence of the integral in (3.27) is absolute.

Proof. Let $(\varepsilon, \lambda, K)$ be fixed, and let $I(\alpha)$ be the integrand of (2.9). The first step of the proof is to show that $\bar{x}$ can be chosen so that for every choice of $r$ and $R, 0<r<R$, the function $I$ is holomorphic on the set

$$
\Sigma(r, R)=\left\{z \in \mathbb{C}^{L} \mid z_{l}=\zeta_{l}^{x}(\alpha, K), r \leqq \alpha_{l} \leqq R, 0 \leqq x \leqq \bar{x}\right\} .
$$

But this is trivial. The function $I(z), z \in \mathbb{C}^{L}$, is holomorphic except on the sets $\left\{z_{l}=0\right\}$ and $\{C(z)=0\}$. Since, by Lemma 2 (i), the function $\zeta_{l}^{x}$ can vanish only if $\alpha_{l}$ vanishes, the manifolds $\left\{z_{l}=0\right\}$ do not intersect $\Sigma(r, R)$. That $\{C(\mathrm{z})=0\}$ also does not intersect $\Sigma(r, R)$ is immediate from Lemma 2 (ii). Hence, the function $I$ is holomorphic on $\Sigma(r, R)$.

The set $\Sigma(r, R)$ is a topologically $(L+1)$-dimensional chain in $\mathbb{C}^{L}$, a fact that allows the application of the Cauchy-Poincaré theorem [14]:

$$
0=F_{\varepsilon, r, R}-\tilde{F}_{\varepsilon, r, R}+\text { (remainder) . }
$$

The remainder comes from the parts of the boundary $\partial \Sigma(r, R)$ on which $\alpha_{l}=r$ or $\alpha_{l}=R$ for some $l$. The task now is to prove that this remainder vanishes in the limit (3.27). This is done by computing integrable upper bounds for $I$ and for the Jacobians appropriate to the surfaces $\alpha_{l}=r$ and $\alpha_{l}=R$.

Consider first the Jacobians $J_{l}$ for the surfaces of constant $\alpha_{l}$. They are, up to an unimportant sign, the determinants of the matrices formed by substituting the column

$$
J_{m 0}=\frac{\partial \zeta_{m}^{x}}{\partial x}=i \zeta_{m}^{x}\left(r_{m}^{2}-\mu_{m}^{2}\right)
$$

for the column $J_{m l}$ of the matrix (3.24). Consider $J_{l}$ on each sector $A^{P}$ separately, expressing it in terms of the sector coordinates (3.9). According to Lemma 1, the functions $J_{l}$ are $C^{\infty}$ in all variables and have the form

$$
J_{l}=t_{L} \tilde{J}_{l}(x, \hat{t}, K) \text {. }
$$


where $\tilde{J}_{l}$ is holomorphic. There exists, therefore, a finite number $m_{0}$, dependent only on $\bar{x}$ and $\boldsymbol{S}^{r}$, such that

for all $l$.

$$
\left|J_{l}\right| \leqq m_{0}|\alpha|
$$

The calculation for $I\left(\zeta^{x}(\alpha, K)\right)$ is similar. The functions are considered on each sector $A^{P}$, sector coordinates (3.9) introduced, and uniform bounds computed. The result is the existence of constants $m_{1}$ and $m_{2}$, dependent only on $\bar{x}, \Lambda_{0}$, and $\mathfrak{R}^{r}$, such that

$$
|I| \leqq m_{1}(1+|\alpha|)^{m_{2}}\left(\prod_{\mathscr{L}} \alpha_{l}^{n_{l}}\right) \exp \left[-\varepsilon \sum_{\mathscr{L}} \alpha_{l} \operatorname{Re} \hat{\zeta}_{l}^{x}\right] .
$$

The exponents $n_{l}$ are given by

$$
n_{l}=\operatorname{Re} \lambda_{l}-1-(\sigma+2)\left(N_{P, P^{-1}(l)}-N_{P, P^{-1}(l-1)}\right),
$$

where $P^{-1}$ denotes the inverse permutation of $P$, and $N_{P, P^{-1}(0)}=0$. They are bounded for $\lambda \in \Lambda_{0}$ and satisfy the inequality $n_{l} \geqq 1$. The quantities $\operatorname{Re} \hat{\zeta}_{l}^{x}$ are, according to the arguments of Lemma 2, bounded from below by a positive number $\varepsilon_{1}$ if $\bar{x}$ is chosen small enough. Consequently, the integrals for the surfaces $\alpha_{l}=R$ are dominated by a factor $\exp \left(-\varepsilon \varepsilon_{1} R\right)$, and those for the surfaces $\alpha_{l}=r$ by a factor $r^{n_{l}}$. Both types of integrals vanish in the limit (3.27), so the remainder in (3.29) can be ignored. Uniform convergence in $(x, \lambda, K)$ is immediate from (3.33). q.e.d.

The homogeneity in $\alpha$ of the integrand of (3.27) can be exploited to obtain a more convenient from of the integral. Let $K$ be any point of $\Omega^{r}$ and $\lambda$ any point of $\Lambda_{0}$. Let $Z_{v}\left(\zeta^{x}, K\right)$ denote the homogeneous (in $\zeta^{x}$ ) components of the polynomial $Z$, the numbers $v$ being the degree of homogeneity. Let $\bar{v}$ be the maximum value of $v$ for which $Z_{v}$ is nonzero. Then the integration over $|\alpha|$ can be performed, leaving only the angular integration over

$$
S^{+}=\left\{\alpha \in \mathbb{R}^{L}|| \alpha \mid=1 ; \alpha_{m} \geqq 0,1 \leqq m \leqq L\right\} .
$$

The resulting expression for the distribution $F(\lambda, K)$ is

where

$$
F(\lambda, K)=\int_{S^{+}} d \alpha \omega(\alpha ; \lambda, K)[\tilde{Y}(\alpha, K)+i 0]^{-\mu(\lambda)},
$$

$$
\begin{aligned}
\omega(\alpha ; \lambda, K)=\frac{\partial\left(\zeta^{x}\right)}{\partial(\alpha)} \sum_{v=0}^{\bar{v}} i^{\mu(\lambda)-p(v)} \Gamma(\mu(\lambda)-p(v)) \\
\cdot Z_{v}\left(\zeta^{x}, K\right) W\left(\zeta^{x}, \lambda\right)\left[Y\left(\zeta^{x}, K\right)\right]^{p(v)}, \\
\tilde{Y}(\alpha, K)=Y\left(\zeta^{x}, K\right), \\
\mu(\lambda)=\sum \lambda_{l}+\bar{v}, \\
p(v)=(\sigma+2)(L-V)+\bar{v}-v .
\end{aligned}
$$

Eq. (3.36) is used in the sequel as the standard expression for $F(\lambda, K)$. 


\section{Non-Landau Points}

The first problem is to reproduce the well known property that the renormalized Feynman integrals are singular in $\mathbb{E}$ only on the positive- $\alpha$ Landau surfaces ${ }^{5}$. These surfaces are associated with the Landau equations which, for the graph $G$, are usually written

$$
\begin{aligned}
\sum_{\mathscr{L}} e(r, l) p_{l}+q_{r}(K) & =0, \quad(r \in \mathscr{V}), \\
\sum_{\mathscr{L}} \eta_{l i} \alpha_{l} \mathrm{p}_{l} & =0, \quad(1 \leqq i \leqq L-V), \\
\alpha_{l}\left(p_{l}^{2}-\mu_{l}^{2}\right) & =0, \quad(l \in \mathscr{L}) .
\end{aligned}
$$

The matrix $[\eta]$ in $(4.1$ b) is the so-called loop matrix; it is any $L \times(L-V)$ matrix, the columns of which span the null space of the incidence matrix $[e]$. The positive- $\alpha$ requirements are expressed in the form

$$
\alpha \in S^{+}, \quad K \in \mathbb{E}^{r} .
$$

The positive- $\alpha$ surface $\mathfrak{L}^{+}\{G\}$ is the set of all $K \in \mathbb{E}^{r}$ for which Eqs. (4.1) have a solution. The desired result is the following theorem:

Theorem 2. Let $G$ be a Feynman graph, and let $F(K)$ be the corresponding renormalized Feynman integral.

(a) If $\bar{K}$ belongs to $\mathbb{E}^{-}-\mathfrak{Q}^{+}\{G\}$, there is a complex neighborhood $\mathfrak{\Re} \subset \vec{E}$ of $\bar{K}$ on which $F(K)$ is holomorphic.

(b) If $\bar{K}$ belongs to $\mathfrak{P}-\mathfrak{I}^{+}\{G\}$, there is a complex neighborhood $\mathfrak{N} \subset \mathfrak{B}$ of $\bar{K}$ on which the mass shell restriction of $F(K)$ is holomorphic.

Proof. Part (b) is an immediate consequence of (a) since $\mathfrak{B}$ is a submanifold of $\mathfrak{E}$.

To prove (a) let the original contour of integration be deformed in accordance with Theorem 1, assuming that $\mathfrak{\Omega}^{r}$ contains $\bar{K}$ as an interior point. This means that (3.36), with $x$ fixed, can be used. The domain of integration $S^{+}$is divided into sectors $S^{+, P}=S^{+} \cap A^{P}$. Sector coordinates (3.9) are introduced, the variable $t_{L}$ being replaced by

$$
\left\lceil 1+\sum_{l=1}^{L-1}\left(t_{L-1} \ldots t_{1}\right)^{2}\right]^{-\frac{1}{2}} \text {. }
$$

The lemmata of Section III imply that all of the functions appearing in the integrand of (3.36) are holomorphic in $(\hat{t}, K)$ at every point of $\hat{T} \otimes \mathfrak{S}^{r}$. The distribution $F(\lambda, K)$ is therefore a holomorphic function at $\bar{K}$, provided that $\tilde{Y}(\alpha, K)$ does not vanish on any sector $S^{+, P}$. It is trivial,

${ }^{5}$ Although this is a widely accepted fact, the author has found only one published reference that treats arbitrary renormalized integrals, a remark in Section 4 of the paper of $[11]$. 
given Lemma 3, that this can happen only if $\bar{K}$ belongs to $\mathfrak{L}^{+}\{G\}$. (The vectors $r_{l}$ defined by (2.14) automatically satisfy (4.1 a) and (4.1 b).)

Thus, if $K$ does not belong to $\mathfrak{I}^{+}\{G\}$, it follows that there is a complex neighborhood $\mathfrak{i} \subset \mathfrak{E}, \mathfrak{R} \cap \mathbb{E}^{r} \subset \mathfrak{S}^{r}$, of $\bar{K}$ on which $F(\lambda, K)$ is holomorphic. Meromorphic continuation in $\lambda$ of the type described by (2.16) follows in the same way as in [3]: the integral is divided into integrals over sectors $S^{+, P}$, sector coordinates introduced, and partial $t$-integrations performed. That this leads to the desired results is insured by Lemma 2 . The holomorphy of $F(K)$ then follows from (2.17). q.e.d.

\section{Landau Points}

In the last section it was proved that (2.17) defines a holomorphic function on each connected component of $\mathfrak{P}-\mathfrak{L}^{+}\{G\}$. The question in this section is how the functions in different components are related. When there are no mass shell restrictions, the answer is well known [11]. Set $x=0$ in (3.41). Then it is clear that $F(K)$ can be continued from any real point of holomorphy to any complex point of $\mathbb{E}$ of the form $K=(1+i y) \operatorname{Re} K, y$ real. From this it follows that there is a single holomorphic function such that its boundary values on $\mathbb{E}^{r}$ form the distribution $F(K)$. Unfortunately, this "ic-prescription" is generally incompatible with mass shell requirements, and another answer to the question must be sought.

As a first step in obtaining this answer a more elaborate classification of points in $\mathfrak{L}^{+}\{G\}$ is introduced. It is here that the directions of the lines of the graph $G$ become important. Mathematically they are included by enforcing the extra condition

$$
\alpha_{l} p_{l}^{0} \geqq 0, \quad(l \in \mathscr{L}) .
$$

This leads to the following definitions:

$\mathfrak{Q}^{+}[G]$ : the subset of $\mathfrak{Q}^{+}\{G\}$ on which (4.1) and (5.1) are satisfied.

$\mathfrak{I}_{1}^{+}[G]$ : the subset of $\mathfrak{L}^{+}[G]$ corresponding to solutions with $\alpha$ in the interior of $S^{+}$.

$\mathfrak{Q}_{0}^{+}[G]$ : the set of all points of $\mathfrak{Q}_{1}^{+}[G]$ that do not belong to $\mathfrak{I}_{1}^{+}\left[G^{\prime}\right]$ for any $G^{\prime} \subset G$.

The set $\mathfrak{L}^{+}\{G\}$ is then the union of all sets $\mathfrak{Q}_{0}^{+}\left[G^{\prime}\right], G^{\prime} \in\{G\}$.

Suppose now that $\bar{K}$ belongs to $\mathfrak{L}^{+}\{G\}$. Then there is a finite set of graphs $G_{g}$, indexed by a set $\mathscr{I}(\bar{K})$, with the following properties:

(i) Each graph $G_{q}$ belongs to $\{G\}$.

(ii) The point $\bar{K}$ belongs to the closure of each of the sets $\mathfrak{I}_{1}^{+}\left[G_{q}\right]$.

(iii) If $\bar{K}$ belongs to the closure of $\mathfrak{I}_{1}^{+}\left[G^{\prime}\right], G^{\prime} \in\{G\}$, then $G^{\prime} \leqq G_{g}$ for some $g$. 
(iv) None of the graphs $G_{g}$ are contractions of any of the other graphs $G_{g^{\prime}}$.

For each $g \in \mathscr{I}(\bar{K})$, there is a further index set $\mathscr{I}_{g}(\bar{K})$. This set labels contractions $G_{h} \cong G_{g}$ for which $\bar{K} \in \mathfrak{Q}_{0}^{+}\left[G_{h}\right]$.

The sets $\mathfrak{Q}_{0}^{+}\left[G_{h}\right]$ have one very important property. They are real analytic submanifolds of $\mathfrak{E}^{r}$ of codimension 1 [7]. That is, if $\bar{K} \in \mathfrak{R}_{0}^{+}\left[G_{h}\right]$, there are a neighborhood $\Omega^{r} \subset \mathbb{E}^{r}$ of $\bar{K}$ and a real analytic function $\Phi_{h}(K)$, with gradient $\nabla \Phi_{h}(K)$ that does not vanish on $\mathfrak{R}^{r}$, such that

$$
\mathfrak{S}^{r} \cap \mathfrak{R}_{0}^{+}\left[G_{h}\right]=\left\{K \mid K \in \mathfrak{S}^{r}, \Phi_{h}(K)=0\right\} .
$$

As is obvious from the Landau equations (4.1), the function $\Phi_{h}$ depends on $K$ only through the combinations $q_{r}(K), r \in \mathscr{V}_{0}$. That is, there is a real analytic function $X_{h}(Q), Q=\left(q_{1}, q_{2}, \ldots, q_{v}\right)$, such that

$$
\Phi_{h}(K)=X_{h}(Q(K)) .
$$

According to Eqs. (4.4), (E.5), and (E.45) of [7] the sign of $X_{h}$, and hence of $\Phi_{h}$, can be chosen so that

$$
c_{h} \sum_{\mathscr{V}} e(r, l)\left(\frac{\partial X_{h}}{\partial q_{r v}}\right)(Q(\bar{K}))=\alpha_{l} p_{l}^{v}, \quad(l \in \mathscr{L}) .
$$

The number $c_{h}$ is positive and $\left\{\alpha_{l}, p_{l}\right\}$ is the solution to (4.1) corresponding to $G_{h}$.

The functions $\Phi_{h}$ are now used to define other functions $\phi_{h}$ :

$$
\phi_{h}(K, \theta)=\operatorname{Im}\left(K \cdot \nabla \Phi_{h}(\bar{K})\right)-\|\operatorname{Im} K\|\left\|\nabla \Phi_{h}(\bar{K})\right\| \cos \theta,
$$

where $K \in \mathfrak{E}$ and $\theta$ belongs to the open interval $\left(0, \frac{\pi}{2}\right)$. In (5.5) the notation

$$
\begin{aligned}
K \cdot \nabla \Phi_{h} & =\sum_{\mathscr{L}_{0}} \sum_{v=0}^{3} k_{j v}\left[\nabla \Phi_{h}\right]_{j}^{v}, \\
{\left[\nabla \Phi_{h}\right]_{j}^{v} } & =\frac{\partial \Phi_{h}}{\partial k_{j v}},
\end{aligned}
$$

is adopted. The functions $\phi_{h}$ are used, in turn, to define the sets

$$
\mathfrak{I}_{g}(\theta)=\left\{K \in \mathfrak{E} \mid \phi_{h}(K, \theta)>0, h \in \mathscr{I}_{g}(\bar{K})\right\} .
$$

It is always presumed in the sequel that $\theta$ is chosen so that all of the sets $\mathfrak{I}_{g}(\theta)$ are nonempty. That this is possible follows from (5.4): this equation implies that $\bar{K} \cdot \nabla \Phi_{h}(\bar{K})>0$ for all $h$ and hence that all the vectors $\nabla \Phi_{h}$ lie in an open half space. Consequently, all of the sets are nonempty if $\theta$ is chosen close enough to $\pi / 2$. 
A theorem that leads to a partial answer to the problem of the $i \varepsilon$-prescription can now be stated. It is the exact analog in $\mathbb{E}$ of Theorem 4 of $[7]$.

Theorem 3. Let $G$ be a connected Feynman graph, and let $F(K)$ be the corresponding renormalized Feynman integral. Let $\bar{K}$ belong to $\mathfrak{L}^{+}\{G\}$. Then there exist $\theta$ in the open interval $(0, \pi / 2)$ and a complex neighborhood

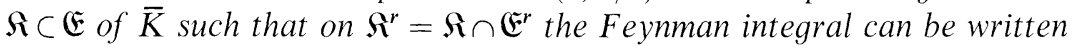
as a sum

$$
F(K)=\sum F^{g}(K) .
$$

The sum is over $\mathscr{I}(\bar{K})$, and the objects $F^{g}$ are Schwartz distributions that are boundary values of functions holomorphic on the (nonempty) sets $\mathfrak{i} \cap \mathfrak{I}_{g}(\theta)$. The boundary values are themselves holomorphic on $\mathfrak{S}^{r}-\mathfrak{I}^{+}\{G\}$.

Proof. The notation is the same as in previous sections. The compact convex set $\boldsymbol{\Omega}_{0}$ of Section III is chosen to be

$$
\Omega_{0}=\left\{K \mid K \in \mathfrak{E},\|K-\bar{K}\| \leqq \varrho_{0}\right\} .
$$

The parameter $x$ of the mapping $\zeta^{x}$ is held fixed at a value determined by Theorem 1 and the lemmata of Section III. Further explicit reference to $x$ is supressed.

If $\bar{K}$ belongs to $\mathfrak{Q}^{+}\{G\}$, there is a nonempty set of positive- $\alpha$ solutions $\left\{\alpha_{l}, p_{l}\right\}$ to the Landau equations (4.1). Let the projection of this set on $S^{+}$be $S_{0}$. The subsets of $S_{0}$ corresponding to solutions $\left\{\alpha_{l}, p_{l}\right\}$ associated with the sets $\mathfrak{L}^{+}\left[G_{g}\right], g \in \mathscr{I}(\bar{K})$ are denoted by $S^{g}$. It is worth remarking that it is not known if the sets $S^{g}$ are pairwise disjoint. Their union, of course, is $S_{0}$.

The idea is to introduce into (3.36) a partition of unity with respect to the sets $S^{g}$. This splits, via (2.17), the distribution $F(K)$ into a sum of the form (5.8). The bulk of the proof is devoted to proving that the distributions $F^{g}$ so defined have the required properties.

The constituent functions $\pi^{g}(\alpha)$ of the partition of unity are first defined on the entire unit sphere and then restricted to $S^{+}$.

(i) The functions $\pi^{g}$ are $C^{\infty}$ on the unit sphere and assume values in the closed unit interval $[0,1]$.

(ii) The function $\pi^{g}$ is strictly positive on $S^{g}$ and has support in

$$
S^{g}(\delta)=\left\{\alpha \in \mathbb{R}^{L}|| \alpha\left|=1, \inf _{\beta \in S^{g}}\right| \alpha-\beta \mid<\delta\right\} .
$$

(iii) The inequality $\sum \pi^{g} \leqq 1$ holds for all $\alpha$, with equality holding on

where $0<\delta_{0}<\delta$.

$$
S_{0}\left(\delta_{0}\right)=\cup S^{g}\left(\delta_{0}\right),
$$

(iv) $\pi^{0}=1-\sum \pi^{g}$. 
There are two reasons for defining the functions $\pi^{g}$ on the entire unit sphere. The first is that existence is guaranteed by standard theorems. The second is that it is a simple way to insure $C^{\infty}$ behavior at the boundary of $S^{+}$. This is important because the partial integrations used to prove that the renormalization (2.17) is possible can be carried out separately for each of the distributions

$$
F^{g}(\lambda, K)=\int_{S^{+}} d \alpha \pi^{g}(\alpha) \omega(\alpha ; \lambda, K)[\hat{Y}(\alpha, K)+i 0]^{-\mu(\lambda)} .
$$

It is sufficient, therefore, to prove the theorem for the distributions (5.12) with $\lambda \in \Lambda$, the result then being automatic for the renormalized distributions.

Consider first $F^{0}(\lambda, K)$. Because $\alpha$ is restricted to the support of $\pi^{0}$, and that support is contained in $S^{+}-S_{0}\left(\delta_{0}\right)$, the function $\tilde{Y}(\alpha, K)$ cannot vanish when $K=\bar{K}$. It therefore cannot vanish in some neighborhood of $K$, say in

$$
\Omega_{\varrho}=\{K \in \mathbb{E} \mid\|\mathrm{K}-\bar{K}\|<\varrho\},
$$

where $\varrho<\varrho_{0}$. The holomorphy (in $K$ ) of $\omega$ and $\tilde{Y}$ imply that $F^{0}$ is holomorphic on $\mathfrak{K}_{\varrho}$. It is therefore of no interest for the purposes of the theorem and is considered as absorbed into one of the other distributions $F^{g}$.

Consider next the distributions $F^{g}(\lambda, K)$. Let $K \in \mathfrak{R}_{\varrho}$ and let

$$
K_{y}=\operatorname{Re} K+i y \operatorname{Im} K, \quad(0 \leqq y \leqq 1) .
$$

It is clear from (5.13) that $K_{y} \in \mathfrak{S}_{\varrho}$ for all $y$. The imaginary part of $\tilde{Y}$ at $K$ can be estimated by using the mean value theorem, the required continuity in $y$ being guaranteed by Lemma 2 . The result is

$$
\operatorname{Im} \tilde{Y}(\alpha, K)=\operatorname{Im} \tilde{Y}(\alpha, \operatorname{Re} K)+\operatorname{Re}\left\{(\operatorname{Im} K) \cdot \nabla_{K} \tilde{Y}\left(\alpha, K_{\bar{y}}\right)\right\},
$$

where $\bar{y} \in[0,1]$. Only those points $\alpha$ in the support of $\pi^{g}$ are of interest in (5.15), and these are contained in $S^{g}(\delta)$. There is, therefore, a point $\beta \in S^{g}$ such that $|\alpha-\beta|<\delta$. At such a point the equation $\nabla_{K} \tilde{Y}(\alpha, K)$ $=\nabla_{K} Y(\alpha, K)$ is true. Substitution of this information, together with (3.18), into (5.15) yields

$$
\begin{aligned}
\operatorname{Im} \tilde{Y}(\alpha, K) & \geqq(\operatorname{Im} K) \cdot \nabla_{K} Y(\beta, \bar{K}) \\
& +\operatorname{Re}\left\{(\operatorname{Im} K) \cdot \nabla_{K}\left[\tilde{Y}\left(\alpha, K_{\bar{y}}\right)-\tilde{Y}(\beta, \bar{K})\right]\right\} .
\end{aligned}
$$

Lemma 3 implies that on $\mathfrak{S}_{\varrho}$ the right side of (5.16) is bounded from below by

$$
h_{g}(K, \bar{K})=\inf _{\beta \in S^{g}}\left\{(\operatorname{Im} K) \cdot \nabla_{K} Y(\beta, \bar{K})-\|\operatorname{Im} K\| M(\varrho+\delta)\right\} .
$$


Therefore, for any $K$ in the set

$$
\mathfrak{S}_{\varrho, g}=\left\{K \in \mathfrak{S}_{\varrho} \mid h_{g}(K, \bar{K})>0\right\}
$$

the integral (5.12) defines a function $H^{g}(\lambda, K)$ that is holomorphic.

The next step is to prove that the numbers $\varrho$ and $\delta$ can be chosen small enough that $\mathfrak{I}_{g}(\theta) \cap \mathfrak{\Omega}_{\underline{o}} \cong \mathfrak{\Omega}_{\varrho, g}$. The key to the proof is the observation that all of the functions depend on $K$ through the combinations $q_{r}(K)$ :

$$
Y(\alpha, K)=Y^{\prime}(\alpha, Q(K)) .
$$

Explicit computation reveals that

$$
\sum_{\mathscr{\gamma}} e(r, l)\left(\frac{\partial Y^{\prime}}{\partial q_{r v}}\right)(\alpha, Q(\bar{K}))=2 \alpha_{l} r_{l}^{v}(\alpha, \bar{K}), \quad(l \in \mathscr{L}) .
$$

This means, according to Theorem 8 of [7], that

$$
\nabla_{Q} Y^{\prime}(\alpha, Q(\bar{K}))=\sum_{\mathscr{g}_{g}(\bar{K})} c_{h} \nabla_{Q} X_{h}(Q(\bar{K})), \quad c_{h} \geqq 0,
$$

when $\alpha \in S^{g}$. It now follows immediately from

$$
(\operatorname{Im} K) \cdot \nabla_{K} Y(\alpha, K)=(\operatorname{Im} Q(K)) \cdot \nabla_{Q} Y^{\prime}(\alpha, Q(K))
$$

and the triangle inequality that $\mathfrak{J}_{g}(\theta) \cap \mathfrak{\Omega}_{\varrho} \cong \mathfrak{\Re}_{\varrho, g}$ if

$$
(\delta+\varrho)<M^{-1}\left\{\inf _{x \in S^{q}}\left\|\nabla_{K} Y(\alpha, \bar{K})\right\|\right\} \cos \theta .
$$

The possibility that $\left\|\nabla_{K} Y(\alpha, \bar{K})\right\|$ vanishes in $S^{g}$, and hence that (5.23) cannot be satisfied with nonzero $\varrho$ and $\delta$, is precluded by (5.20). Hence, the set $\mathfrak{I}_{g}(\theta)$ satisfies $\mathfrak{I}_{g}(\theta) \cap \mathfrak{I}_{Q} \leqq \mathfrak{I}_{Q, g}$ and $H^{g}$ is holomorphic on $\mathfrak{J}_{g}(\theta) \cap \mathfrak{S}_{\underline{\underline{Q}}}$.

The proof is completed by a discussion of what is meant by boundary value. Let $K_{g}(y, K)$ be a $C^{\infty}$ mapping from $[0,1] \otimes\left(\Omega_{e} \cap \mathbb{E}^{r}\right)$ into $\boldsymbol{\Omega}_{e}$ with the properties $K_{g}(0, K)=K$ and $K_{g}(y, K) \in\left(\mathfrak{J}_{g}(\theta) \cap \boldsymbol{\Re}_{0}\right)$ for all $y>0$. It is easy to see that such mappings exist. (Consider the function $K+i y \bar{K}$.) Let $f(K)$ be a Schwartz test function with support in $\mathfrak{K}^{r}=\mathfrak{S}_{\varrho} \cap \mathbb{E}^{r}$, and consider

$$
\lim _{r \rightarrow 0^{+}} \int_{\Omega^{r}} d K f(K) H^{g}\left(\lambda, K_{g}(y, K)\right), \quad(g \in \mathscr{I}(\bar{K})) .
$$

The standard technique of partial integration, say with respect to the local coordinate $\|K\|$, shows that the limit exists and is equal to

$$
\int_{\Omega^{r}} d K f(K) F^{g}(\lambda, K), \quad(g \in \mathscr{I}(\bar{K})) .
$$


Thus, the original distributions $F^{g}(\lambda, K)$ are boundary values, in the sense of (5.24), of the holomorphic functions $H^{g}(\lambda, K)$. It is obvious from (5.12) that $F^{g}(\lambda, K)$ is holomorphic at points of $\mathfrak{R}^{r}-\mathfrak{L}^{+}\{G\}$, thus completing the proof. q.e.d.

The major problem in proving a mass shell theorem analogous to Theorem 3 is in showing that the sets $\mathfrak{J}_{g}(\theta)$ have nonempty intersections with $\mathfrak{W}$. For this an additional technical assumption appears to be necessary.

Theorem 4. Let $G$ be a connected Feynman graph, and let $F(K)$ be the corresponding renormalized Feynman integral, considered as a distribution on $\mathfrak{P}$. Let $\bar{K}$ belong to $\mathfrak{P} \cap \mathfrak{Q}^{+}\{G\}$. Suppose further that:

All graphs $G_{g}, g \in \mathscr{I}(\bar{K})$, are stable particle graphs on $\mathfrak{P}$.

Then there exists $\theta$ in the open interval $(0, \pi / 2)$ and a complex neighborhood $\mathfrak{N} \subset \mathfrak{B B}$ of $\bar{K}$ such that on $\mathfrak{N}^{r}=\mathfrak{B} \cap \mathfrak{N}$ the renormalized integrals $F^{g}(K)$ of Theorem 3 are the boundary values of functions holomorphic on the (nonempty) sets $\mathfrak{R} \cap \mathfrak{I}_{g}(\theta)$.

Proof. It is sufficient to prove that the set $\mathfrak{N} \cap \mathfrak{I}_{g}(\theta)$ is nonempty and has $\mathfrak{N}^{r}$ as part of its boundary. Let $z$ denote the local mass shell coordinates, with $\bar{K}=K(\bar{z})$. Let $\mathfrak{N}=\mathfrak{B} \cap \mathfrak{R}_{\varrho}$ with $\boldsymbol{\Omega}_{\varrho}$ defined by (5.12) and $\varrho$ chosen small enough that $\mathfrak{N}$ is contained in a single coordinate patch. Let $K_{r}$ be any real point in $\mathfrak{R}^{r}$ with corresponding local coordinate $z_{r}$. Let $z_{u}=(1-u) z_{r}+u z$, where $z$ is some point such that $K_{u}=K\left(z_{u}\right)$ belongs to $\mathfrak{N}$ for all $u, 0 \leqq u \leqq 1$. The functions $\phi_{h}(u)=\phi_{h}\left(K_{u}, \theta\right)$ are $C^{\infty}$ in $u$ and vanish at $u=0$. Hence, if the first derivative at $u=0$ is positive, there is a semi-closed interval $(0, \bar{u}]$ on which $\phi_{h}(u)$ is positive. Define $d_{h}$ to be

$$
d_{h}=\operatorname{Im}\left\{\left.\left(z-z_{r}\right) \cdot \frac{\partial(K)}{\partial(z)}\right|_{\bar{z}} \cdot \nabla \Phi_{h}(\bar{K})\right\} .
$$

The difference between $\phi_{n}^{\prime}(0)$ and $d_{n}$ can be estimated with the help of the continuity of $K(z)$. This implies that there is a positive finite constant $c$ such that

$$
\left|\phi_{h}^{\prime}(0)-d_{h}\right| \leqq c\left\|\nabla \Phi_{h}(\bar{K})\right\|\left|\operatorname{Im}\left(z-z_{r}\right)\right|\left(\cos \theta+\left|\bar{z}-z_{r}\right|\right) .
$$

If

$$
U_{h}=\left.\frac{\partial(K)}{\partial(z)}\right|_{\bar{z}} \cdot \nabla \Phi_{h}(\bar{K})
$$

is nonzero, then $z$ can be chosen so that $d_{h}$ is positive. It is then clear from (5.28) that if $\theta$ is chosen close enough to $\pi / 2$ and if $\varrho$ is chosen small 
enough (so that $\left|z-z_{r}\right|$ and $\left|z_{r}-\bar{z}\right|$ are sufficiently small), then $\phi_{h}^{\prime}(0)$ is positive. Of course, the same choice of $z$ must suffice for all $h \in \mathscr{I}_{g}(\bar{K})$.

The idea is to prove that all of the vectors $U_{h}$ lie in an open half space. Suppose not. Then there exist constants $c_{h}$ such that

$$
\begin{aligned}
\sum c_{h} U_{h} & =0, \\
\sum c_{h} & =1, \quad c_{h} \geqq 0 .
\end{aligned}
$$

This implies (Eq. (B.10) of [7]) that

where

$$
\sum c_{h} \nabla \Phi_{h}(\bar{K})=U_{0},
$$

$$
U_{0}=\left(a+b_{1} \bar{k}_{1}, \ldots, a+b_{n} \bar{k}_{n}\right) .
$$

The vector $a$ is arbitrary and the quantities $b_{j}$ are scalars. The left side of (5.31) depends, however, on $\bar{K}$ only through the combination $Q(\bar{K})$. This means that if the external particle $j$ is an initial particle and is attached to the same vertex as another initial particle $i$, then $b_{i}=b_{j}=0$. This is because $U_{0 l}=U_{0 j}$, while $\bar{k}_{i}$ and $\bar{k}_{j}$ are not collinear $(\bar{K} \in \mathfrak{P})$. Similar conclusions hold for final particles. If $G_{g}$ is a stable particle graph, there must be some "initial" vertex $v_{i}$ with at least two initial external particles, and a "final" vertex $v_{f}$, positive timelike with respect to $v_{i}$, with at least two final particles. That is, there are two vertices $i$ and $f$, each with two external particles attached, such that they are connected by a chain of lines, all of which are oriented in the direction $i$ to $f$. It follows from (5.3) that $c_{h}=0$ in (5.31) unless the two vertices $i$ and $f$ coincide in $G_{h}, G_{h} \subseteq G_{g}$. The same arguments are now applied to these remaining diagrams. A finite induction leads to $c_{h}=0$ for all $h$. This contradicts (5.30) so the hypothesis that the vectors $U_{h}$ are not confined to an open half space is wrong ${ }^{6}$. It follows that $z$ can be chosen so that $\phi_{h}^{\prime}(0)>0$ for all $h$, with the consequence that $\mathfrak{N} \cap \mathfrak{J}_{g}(\theta)$ is nonempty. The fact that $\mathfrak{N}^{r}$ lies on the boundary of $\mathfrak{N} \cap \mathfrak{I}_{g}(\theta)$ is a consequence of (5.27) and (5.28). q.e.d.

At first glance (5.26) is not objectionable, at least from the $S$-matrix point of view. It is, after all, only the stable particle graphs that are of interest in $S$-matrix theory.

There are, however, a number of objectionable features. First, there is no known rule for deciding if (5.26) is actually satisfied. In fact, if the graphs $G_{g}, g \in \mathscr{I}(\bar{K})$ are not all contractions of the same stable particle graph $G^{\prime} \in\{G\}$, it is unlikely that the stability condition (5.26) can be enforced. Second, except in the accidental case $\mathfrak{P} \subset \mathfrak{Q}^{+}\{G\}$, the assumption (5.26) is superfluous for almost all $\bar{K}$. This is because the set of points $\bar{K}$ at which the sets $\mathfrak{B} \cap \mathfrak{Q}_{0}^{+}\left[G_{h}\right], h \in \cup \mathscr{I}_{g}(\bar{K})$, are not manifolds is of

6 This paragraph corrects an error in the proof of Theorem 13 of [7]. The error is that the second of Eqs. (E.60) of [7] does not necessarily follow from the first. 
(Lebesgue) measure zero with respect to $\mathfrak{B} \cap \mathfrak{I}_{0}^{+}\left[G_{h}\right]$. Thus either two of the sets $\mathfrak{B} \cap \mathfrak{Q}_{0}^{+}\left[G_{h}\right]$ coincide or they intersect in a set of zero measure. The first case cannot lead to conflicting $i \varepsilon$-prescriptions [7] and so can be ignored. In the second case the set of points $\bar{K}$ for which $\mathscr{I}(\bar{K})$ has more than one member is of measure zero, proving the assertion that (5.26) is superfluous almost everywhere. Third, a violation of (5.26) necessitates such bizarre circumstances that it is hard to imagine that such a situation is possible. The simplest violation is the one in which there are two graphs $G_{1}, G_{2} \in\{G\}$ such that $\bar{K} \in\left(\mathfrak{Q}_{0}^{+}\left[G_{1}\right] \cap \mathfrak{Q}_{0}^{+}\left[G_{2}\right]\right)$. The graphs have some lines in common, at least one of which is oriented differently in $G_{1}$ than in $G_{2}$. This means that at $\bar{K}$ the sign of the energy of the corresponding internal particle is indeterminant! No examples of this phenomenon are known.

What are the possibilities if (5.26) is not generally valid? There is, first of all, at least one class of graphs for which the assumption is not necessary. This class includes all single loop graphs that can be stable particle graphs at all.

Theorem 5. Let $G$ be a connected Feynman graph that has at least two external particles at each vertex. Let $\bar{K}$ belong to $\mathfrak{B} \cap \mathfrak{I}^{+}\{G\}$, and suppose that no two vectors $\bar{k}_{j}, \bar{K}=\left(\bar{k}_{1}, \ldots, \bar{k}_{n}\right)$, are collinear. Then Theorem 4 is true at $\bar{K}$ without the assumptions (5.26).

Proof. At such a point $\bar{K}$ it follows (from the argument following (5.32)) that $U_{0}=0$ in (5.31). Eq. (5.7) implies, on the other hand, that $\bar{K} \cdot \nabla \Phi_{h}(\bar{K})>0$ for all $h$. This implies that all of the constants $c_{h}$ in (5.31) must vanish. The argument now proceeds as for Theorem 4. q.e.d.

Another possibility is to find an alternative method of proof of Theorem 4 that does not require particle stability. No progress in this direction has been made.

The only other viable possibility seems to be to prove that (5.26) is true if only one of the graphs $G_{g}, g \in \mathscr{I}(K)$, is a stable particle graph. No such proof is known at present.

There remains the problem associated with the independence property. No information is provided in Theorem 4 about the division of singularities among the distributions $F^{g}$. The independence property is equivalent to the assertion that $F^{g}$ is singular only on $\mathfrak{L}^{+}\left[G_{g}\right]$. A proof of this would require a proof that the sets $S^{g}$ (defined in the proof of Theorem 3) are pairwise disjoint. No counterexamples are known, but there is also no general proof that it is so.

Acknowledgements. It is a pleasure to thank the Seminar für theoretische Physik of the ETH for their kind hospitality, and the Swiss National Fund for its financial support. The author is particularly indebted to Professors K. Hepp and W. Hunziker and to Dr. M. Simonius for numerous conversations. 


\section{References}

1. Eden, R. J., Landshoff, P. V. Olive, D. I., Polkinghorne, J. C.: The analytic $S$-matrix. Cambridge: Cambridge Univ. Press 1966.

2. Bjorken, J. D., Drell, S. D.: Relativistic quantum fields. New York: McGraw-Hill Book Co. 1965.

3. Speer, E. R.: J. Math. Phys. 9, 1404 (1968).

4. - Generalized Feynman amplitudes. Princeton: Princeton Univ. Press 1969.

5. Hepp, K.: Commun. Math. Phys. 14, 67 (1969).

6. Caianello, E. R., Marinaro, M., Guerra, F.: Nuovo Cimento 60 A, 713 (1969). Guerra, F., Marinaro: Nuovo Cimento 60 A, 756 (1969).

7. Chandler, C., Stapp, H. P.: J. Math. Phys. 10, 826 (1969).

8. Iagolnitzer, D., Stapp, H. P.: Commun. Math. Phys. 14, 15 (1969).

9. Chandler, C.: Helv. Phys. Acta 42, 759 (1969).

10. Coster, J., Stapp, H. P.: Lawrence Radiation Laboratory Report UCRL-18512.

11. Hepp, K.: Commun. Math. Phys. 2, 301 (1966).

- Théorie de la renormalisation. Berlin-Heidelberg-New York: Springer 1969.

12. Pham, F.: Introduction à l'étude topologique des singularités de Landau. Paris: Gauthier-Villars 1967.

13. Boyling, J. B.: Nuovo Cimento 53 A, 351 (1968).

14. Fuks, B. A.: Analytic functions of several complex variables. American Mathematical Society, Providence, R.I., p. 264. 1963

Colston Chandler

Seminar für Theoretische Physik der ETH

CH-8044 Zürich, Hochstraße 60

C. Chandler's present address:

Department of Physics and Astronomy

The University of New Mexico

Albuquerque, New Mexico 87 106, USA 